\title{
COARCTATION OF THE AORTA
}

BY

\author{
M. McGREGOR AND M. MEDALIE*
}

From the Cardio-Pulmonary Research Unit (Council for Scientific and Industrial Research) of the Department of Medicine, University of the Witwatersrand and Johannesburg General Hospital

Several anomalies of the great vessels have been described in association with coarctation of the aorta. The case presented below had unusual features which seem worthy of placing on record. In addition, a technique of simultaneous angiocardiography and retrograde aortography is described.

\section{Case Notes}

The patient was an eight-year-old white boy who had always been noted to tire rather easily and to complain of abdominal pain after strenuous exertion. Recently symptoms had increased.

On examination he was thin (height, 54 inches; weight, $47 \mathrm{lb}$.) but was otherwise a normal intelligent child. The most striking feature was the prominence and tortuosity of both carotid vessels which stood out under the skin of the neck. On palpation they were tense and seemed to contain blood at a very high pressure. Elsewhere, there was complete absence of arterial pulsation and the blood pressure could not be ascertained. No scapular or other collateral vessels could be felt.

Cardiac examination showed a heaving apex beat of left ventricular type in the mid-axillary line, and there was slight prominence of the left præcordium. A faint systolic thrill was palpable in the suprasternal notch. On auscultation over the apex, a grade 2 (Levine, 1949) blowing systolic and a grade 4 rumbling mid-diastolic murmur were heard. From the left second to fifth interspaces there was a loud (grade 4) blowing systolic murmur followed by a normally split second sound of average intensity. Over the whole of the skull a fairly loud rumbling continuous murmur typical of arterio-venous aneurysm was present; no point of maximal intensity could be found.

The fundi showed the features of hypertensive retinopathy. The arteries showed irregular zones of contraction and in the right fundus there was some papillœdema with numerous exudates and a macula star. The diastolic blood pressure in the retinal artery was about $200 \mathrm{~mm}$. of mercury. The rest of the physical examination was negative.

The diagnosis of coarctation of the aorta with severe hypertension of the cerebral circuit and much enlargement of the left ventricle could be made on clinical grounds alone. In addition, it seemed probable that both subclavian arteries arose in or below the coarcted segment. The continuous murmur over the head was possibly due to an arteriovenous aneurysm. However, as localization was not possible, it was more likely that the murmur developed in the greatly dilated channels caused by the increased flow in the carotid vessels. In addition, mitral stenosis was suspected.

Investigations. Apart from a mild anæmia (red cells, 3.8 million per cu. mm.) the blood count was normal. The sedimentation rate was $3 \mathrm{~mm}$. in one hour and the Wassermann reaction was negative.

Phonocardiograms confirmed the presence of the murmurs that had been heard, and electrocardiograms showed the pattern of left ventricular strain.

Routine X-ray examinations did not add to the diagnosis but confirmed several points. In the postero-anterior view the aortic knuckle appeared notmal and there was no rib notching. There was much cardiac enlargement affecting chiefly the left ventricle. The pulmonary vessels appeared

* Clinical Assistant in Pædiatrics, Transvaal Memorial Hospital for Children, Johannesburg. 
normal and the lungs seemed normally vascularized. There was evidence of left atrial enlargement, both in the postero-anterior and in the right anterior oblique views. In the latter view an indentation of the posterior wall of the barium filled asophagus confirmed the presence of an anomalous right subclavian artery, while in the left anterior oblique view considerable enlargement of the left ventricle was observed.

In an attempt to learn as much as possible of the vascular anomalies in this case before resorting to surgery, a routine antero-postero angiocardiogram was performed employing the now widely used technique of Robb and Steinberg (1939) with a cassette changer and certain modifications to the syringe previously described (McGregor, 1949 and 1951). As will be seen (Fig. 1A), two dilated and tortuous carotid vessels were seen to be arising from an otherwise normal aortic arch which ended suddenly at the commencement of the descending aorta. No diodrast entered the distal segment and no collateral vessels could be seen.

In view of the failure of the distal segment to fill with diodrast it was impossible to learn the length of the stenosed segment. Accordingly, two days later the patient was anæsthetized and while a cannula was inserted into an arm vein in the usual manner the femoral artery was exposed, and under screen control a large (No. 10) catheter was passed up the abdominal aorta. The patient was placed in the right posterior oblique position and $25 \mathrm{ml} .70$ per cent diodrast followed by $15 \mathrm{ml}$. saline was injected into the arm vein. After four seconds, injection of $15 \mathrm{ml}$. diodrast was started via the catheter inserted into the femoral artery. Exposures were taken throughout at the rate of approximately three per second. The resultant plates showed a normal right heart and pulmonary artery (Fig. 1B). During filling of the systemic circuit (Fig. 1C) a better view of the aortic arch and origin of the carotids was obtained. There was adequate filling of the descending aorta which appeared normal and it could be seen that the coarcted segment was extremely short, rendering an aortic graft unnecessary. The subclavian arteries did not appear to fill from either the upper or lower segments.

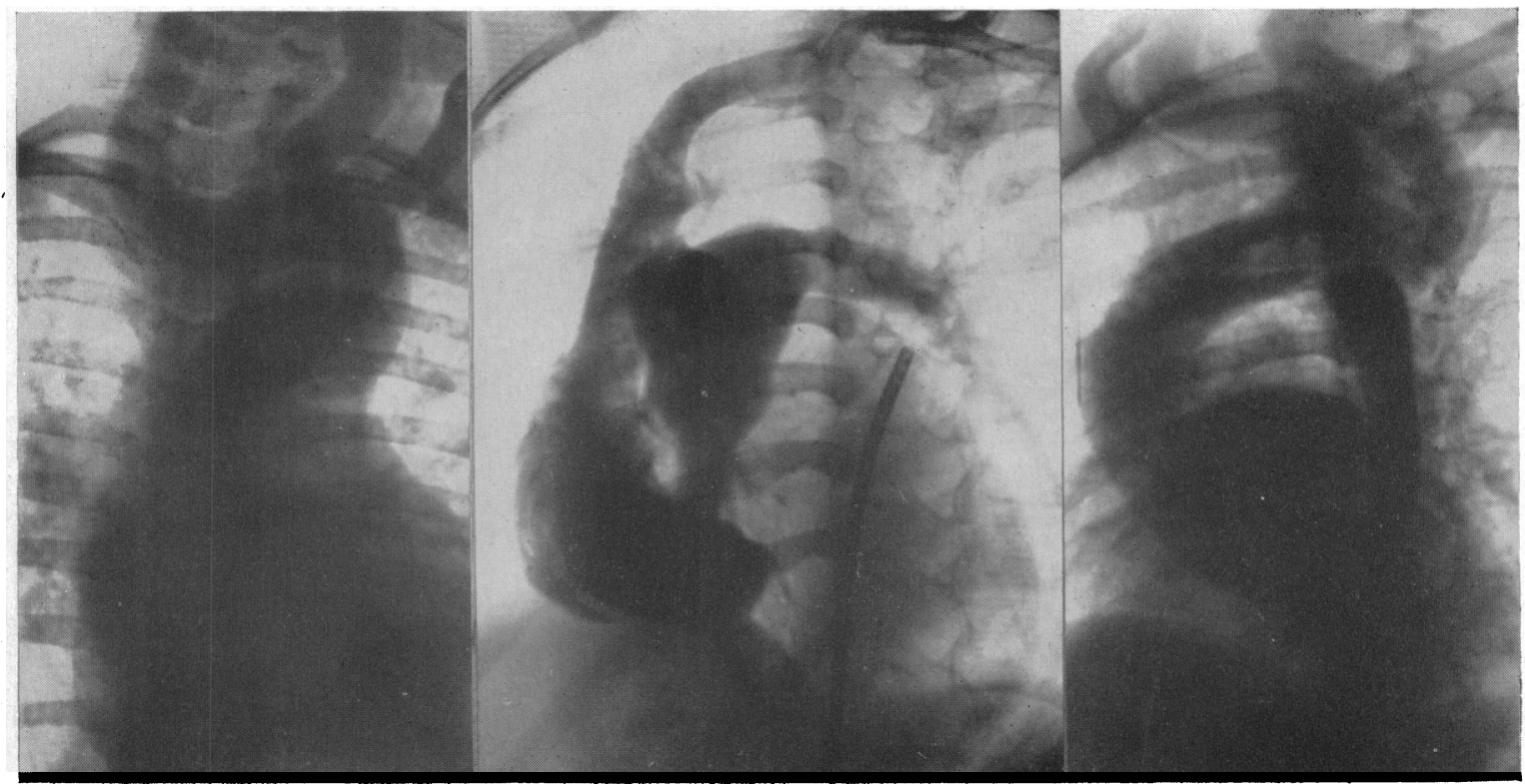

A

B

C

FIG. 1.-Angiocardiograms from this case. (A) After routine angiocardiogram, showing two dilated and tortuous carotid arteries and the absence of filling of the descending aorta. (B) Normal right side of the heart after routine angiocardiography in the right posterior oblique position, also showing a catheter that has been inserted through the femoral artery. (C) The same, but later, showing the left side of the heart: the second injection has been given through the catheter to show the descending aorta (see text). 
Progress. Following the first visit the patient's condition deteriorated rapidly. He developed a partial left facial paralysis, complete loss of vision in the left eye, and early left ventricular failure within the next seven weeks. After digitalization had produced some improvement in condition, surgery was attempted, but the patient died in cardiac arrest during the opening of the thorax.

Post-mortem Summary. Post mortem, an unusually high coarctation of the aorta was found below the insertion of a just patent ductus arteriosus (Fig. 2, F). The aortic arch (A) was dilated but

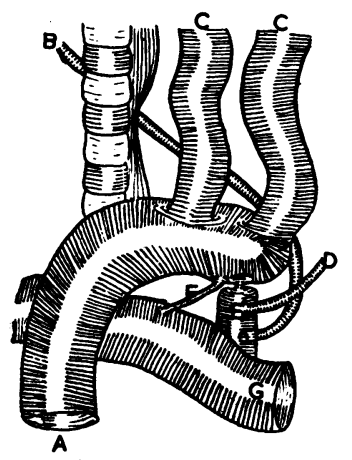
the descending aorta $(E)$ and pulmonary vessels $(G)$ were normal. Both subclavian arteries (B and D) arose distal to the coarctation, the right subclavian being aberrant in course. No dilated intrathoracic collaterals were seen. The carotid vessels (C) showed atherosclerotic changes and there was gross dilatation and atherosclerosis of all the intracerebral vessels. The heart showed dilatation and hypertrophy of the left ventricle, and small vegetations were found along the free edge of the mitral valve though the mitral valve was not actually stenosed. The aortic valve was bicuspid.

FIG. 2.-Diagrammatic representation of the abnormal arteries showing both subclavians arising below the coarctation. (A) Aorta. (CC) Carotid arteries. (B) Right and (D) left subclavian arteries. (E) Descending thoracic aorta. (F) Slightly patent ductus arteriosus. (G) Pulmonary arteries.

\section{Comment}

An anomalous right subclavian artery in association with coarctation of the aorta is extremely rare. In a case reported by Fawcett (1905), and in a post-mortem specimen described by East (1932) the anomalous right subclavian arose proximal to the point of coarctation. In a third case described by Stevens, there was an anomalous left subclavian arising below the coarctation (Gross, 1950.)

The clinical features in our case were striking and were such that the anomaly could be diagnosed with reasonable certainty on clinical grounds alone. It has now become important to define with accuracy the exact length of the coarcted segment. In most cases the length of this can be ascertained from routine angiocardiograms or aortograms via the radial artery, but as in this case, simultaneous angiocardiography and retrograde aortography via the abdominal aorta may sometimes be needed.

\section{Summary}

An unusual case of coarctation of the aorta is described in which both subclavian vessels arose below the site of narrowing. Anomalies of the carotid vessels were also present. Pulses in all four limbs were absent and there was none of the usual evidence of the collateral blood supply to the lower extremities. In order to demonstrate the length of the constricted segment, simultaneous angiocardiography and retrograde aortography was employed.

Our thanks are due to Professor G. A. Elliott and Dr. T. H. Bothwell for helpful criticism in writing this paper. We also wish to acknowledge with gratitude the assistance of our colleagues Dr. J. L. Braudo and Dr. J. Lannon; also Dr. J. Kaye of the Department of Radiology of the Johannesburg Hospital and the members of his staff, and lastly to Dr. R. E. Gross of the Peter Bent Brigham Hospital, Boston, Massachusetts, for his kind assistance and for permission to print the post-mortem findings.

\section{REFERENCES}

East, T. (1932). Proc. Roy. Soc. Med., 25, 797.

Fawcett, J. (1905). Guy's Hosp. Reports, 59, 17.

Gross, R. E. (1950). Circulation, 1, 41.

Levine, S. A., and Harvey, W. P. (1949). Clinical Auscultation of the Heart. W. B. Saunders and Company, London.

McGregor, M. (1949). Brit. J. Radiol., 22, 459.

, (1951). Brit. J. Radiol., 24, 226.

Robb, G. P., and Steinberg, I. (1939). Amer. J. Roentgenol., 41, 1. 